\title{
EVALUACION DE PROYECTOS EN ASIGNATURAS DE AUTOMATIZACIÓN Y ROBÓTICA
}

\author{
David Muñoz de la Peña Sequedo, Francisco Salas Gómez \\ Departamento de Ingeniería de Sistema y Automática \\ dmunoz@us.es, salas@us.es
}

\section{Resumen}

En este trabajo se presenta la implantación de un sistema de evaluación de proyectos en diversas asignaturas de nuestro departamento relacionadas con la automatización y la robótica. La propuesta docente presentada se basa en la realización por parte de los alumnos de un proyecto abierto de automatización en grupo, en el cual no sólo tienen que diseñar la solución, sino también el problema. La naturaleza abierta del proyecto y el gran número de alumnos, motivó el reparto de los diferentes grupos entre los diferentes profesores de la asignatura. Cada grupo de alumnos tiene al menos dos tutorías con su profesor para acordar el problema a resolver para poder adecuar la complejidad y alcance a los objetivos de la asignatura. La evaluación de los proyectos se realiza mediante una presentación oral el mismo día que el examen escrito de las convocatorias oficiales. La metodología propuesta ha sido implantada con éxitos en diversas asignaturas y han participado más de 600 alumnos durante los últimos años con una gran aceptación tanto por parte del profesorado como de los alumnos.

Palabras clave: Evaluación, automatización y robótica industrial.

\section{INTRODUCCIÓN}

Desde hace ya varios años nos encontramos inmersos en la Escuela Técnica Superior de Ingeniería en un proceso de cambio de modelo educativo derivado de la implantación del Espacio Europeo de Educación Superior, creado mediante el proceso de Bolonia. Uno de los principales cambios ha sido la implantación del sistema europeo de transferencia y acumulación de créditos (ECTS) en todos nuestros planes de estudio, una de las principales piedras angulares del proceso de Bolonia pues ofrece un medio adecuado para promover al máximo la movilidad de estudiantes.

Como principal característica, los créditos ECTS se basan en la carga de trabajo necesaria para que los estudiantes alcancen los resultados de aprendizaje esperados, incluyendo el tiempo dedicado a todas las actividades de aprendizaje necesarias como clases, seminarios, proyectos, trabajo práctico, aprendizaje autónomo y exámenes. Como estimación, cada crédito ECTS equivale a entre 25 y 30 horas de trabajo. En nuestra Universidad, los planes de estudio han asignado una carga de 10 horas lectivas por cada crédito de la asignatura, dejando el resto de la carga de trabajo a libre disposición del profesor. En general, el número de horas lectivas ha disminuido en comparación con los planes de estudio anteriores, motivado principalmente porque el sistema de educación propuesto en Bolonia promueve una mayor implicación del alumno en las actividades de aprendizaje y evaluación continua, en contraposición a modelos anteriores basados principalmente en la clase magistral y la evaluación a través de exámenes.

Los recursos docentes sin embargo no se han modificado sustancialmente. En particular, el número de alumnos matriculados y el número de profesores de cada curso son similares a los planes de estudios anteriores, sobre todo en los cursos bajos de los diferentes grados, aunque sí es verdad que en muchas asignaturas de cursos superiores, debido a la mayor optatividad disponible, sí ha bajado el ratio alumnos por profesor. El trabajo necesario para implantar actividades de aprendizaje y evaluación diferentes de la clase magistral y la evaluación por examen, depende en gran medida de los alumnos que un profesor tenga en su asignatura, lo que complica el cambio de modelo educativo.

En este trabajo presentaremos cómo se han abordado los diferentes problemas derivados de la naturaleza de las materias estudiadas, la gestión de grupos masificados y los recursos disponibles para poder incluir dentro de la planificación de la asignatura que los alumnos desarrollen un proyecto de una cierta envergadura. Hay varios problemas para poder incluir este tipo de actividades de aprendizaje en cursos numerosos. En primer lugar el diseño de un proyecto que presente un reto al alumno, con una carga de trabajo apropiada y que cubra los objetivos de aprendizaje de una asignatura es en general muy costoso. En problema con cursos numerosos es que puede ser interesante diseñar más de un proyecto para que los alumnos tengan varias opciones $\mathrm{o}$ incluso tengan cada alumno un proyecto diferente. 
En segundo lugar, la evaluación de un proyecto también puede tener una gran carga de trabajo, que en caso de que la evaluación del proyecto no sustituya a la evaluación convencional de los exámenes, se suma a la carga de trabajo por profesor normal.

Las asignaturas presentadas en este trabajo están centradas en el campo de la automatización y la robótica. Como uno de los principales objetivos de aprendizaje de las mismas se encuentra el que el alumno aprenda a utilizar entornos de programación de dispositivos industriales, como autómatas programables y robots manipuladores. Estos dispositivos tienen un gran coste, y aunque se dispone de diversos equipos de prácticas en los laboratorios, las horas dedicadas en el plan docente a trabajos prácticas limita mucho el uso por parte del alumno. Sin embargo, como alternativa a dispositivos reales y teniendo en cuenta que los objetivos de aprendizaje se centran en el campo de la programación, la idea de poder usar simuladores de estos dispositivos es muy interesante, sobre todo, si es posible que el alumno use dichos simuladores sin necesidad de estar en las instalaciones de la Universidad. En esta línea, hay que decir que los propios fabricantes de autómatas programables y robots manipuladores ofrecen como parte de sus plataformas de desarrollo simuladores de los dispositivos físicos, ofreciendo a menudo licencias de gratuitas, como la plataforma de desarrollo Codesys de la empresa $3 \mathrm{~S}$ o la plataforma Robotstudio de $\mathrm{ABB}$, o con precios especiales para estudiante, como la plataforma Unity Pro de Schneider. Adicionalmente, hay empresas que ofrecen no sólo la simulación de dispositivos de control, sino además de los propios elementos físicos a controlar, es decir, simuladores de dispositivos físicos como la empresa Realgames.

Estos nuevos recursos posibilitan plantear proyectos de diseño de sistemas de automatización de gran interés a los alumnos que puedan resolver fuera de las aulas. En particular, el recurso que habilitó el desarrollo de la experiencia docente actual, es el libro "Introducción a la programación de autómatas programables usando CoDeSys", de Miguel Ángel Ridao Carlini [1]. El objetivo de este libro es proporcionar al lector una introducción a la programación de autómatas programables siguiendo el estándar de la norma IEC-61131-3, utilizando para ello el software de desarrollo de aplicaciones para autómatas programables CodeSys que ofrece la posibilidad de emular un autómata programable y tiene un entorno gráfico sencillo que permite desarrollar simulaciones visuales de sistemas físicos sencillos.
En los próximos apartados describiremos la metodología docente utilizada para incluir un proyecto de diseño y resolución de un sistema de automatización usando Codesys en nuestras asignaturas.

La experiencia docente presentada en este trabajo se implantó por primera vez durante el curso 2014-2015 en la asignatura obligatoria "Automatización y control de sistemas de producción" del Máster Universitario en Ingeniería Industrial y desde entonces forma parte de su programa docente. Posteriormente, teniendo en cuenta la experiencia con los alumnos del máster se implantó en la asignatura "Automatización Industrial" del Grado en Ingeniería de las Tecnologías Industriales desde el curso 2015-2016. Esta asignatura es obligatoria en las intensificaciones "Eléctrica", "Electrónica", "Automática", "Organización y producción". En total más de 600 alumnos han sido evaluados con la metodología propuesta.

\section{OBJETIVOS}

El objetivo principal es conseguir que el alumno aprendiera a diseñar sistemas de automatización con una plataforma industrial siguiendo el estándar de la norma IEC-61131-3 teniendo en cuenta las restricciones de recursos (horas de clase, prácticas y profesores implicados), normativa (tiene que ser compatible con la evaluación ordinaria en convocatorias oficiales) y de número de alumnos. Adicionalmente, un objetivo primordial es que la experiencia fuera repetible en cursos futuros.

Teniendo en cuenta que cada curso de las asignaturas consideradas tienen más de 150 matriculados, nos encontramos con un grave problema para el diseño de los proyectos de automatización, ya que por su naturaleza, sería imposible evitar el plagio de las soluciones de cualquier proyecto. Este problema es intrínseco a las asignaturas de programación, ya que los enunciados de los problemas no son personalizables y es fácil plagiar algoritmos. Por este motivo, se decidió que no se diseñaría un enunciado de un problema para que lo resolvieran los alumnos, sino que la propia definición del problema forma parte del trabajo que tiene que desarrollar los alumnos. Dejar un enunciado libre limita en gran medida el problema del plagio (aunque por supuesto no lo elimina), sin embargo tiene el problema de que los alumnos pueden proponer problemas que no estén de acuerdo con los objetivos de aprendizaje, bien por su alcance o por su dificultad. Esto obliga a incluir un seguimiento parcial de los proyectos, en particular, en la primera fase en la que los alumnos proponen los problemas que resolverán. 
Para que todos los alumnos tengan que realizar el proyecto, uno de los objetivos era que la evaluación pudiera ser compatible con la evaluación mediante exámenes escritos en convocatorias oficiales y con los recursos de profesores disponibles. Esto implica que si el proyecto es obligatorio para aprobar una asignatura, debe de ser posible entregarlo en cualquiera de las tres convocatorias oficiales. La solución propuesta es que los alumnos realicen una presentación oral de los proyectos el mismo día del examen escrito de la convocatoria oficial. Para poder organizar la evaluación, cada profesor evalúa a los grupos a los que tutora. Esto supone una gran implicación por parte del profesorado de la asignatura. A continuación presentamos la metodología utilizada con detalle.

\section{METODOLOGÍA}

La evaluación de la asignatura se realiza en base a un examen escrito (70\% de la nota) y a un proyecto (30\% de la nota). Para aprobar la asignatura es necesario aprobar el examen escrito y el proyecto. La evaluación del proyecto y el examen se realizan conjuntamente el día indicado por el calendario de convocatorias oficiales de la ETSI. La evaluación del proyecto se realiza en base a una presentación de 10 minutos con un turno de preguntas de 5 minutos en la que el alumno debe describir el problema resuelto, la correspondiente solución y el interés del mismo para la asignatura. Los grupos pueden ser de hasta 3 alumnos. La nota del proyecto se guarda para las tres convocatorias oficiales. La nota del examen escrito no se guarda (es necesario hacer el proyecto para poder realizar el examen).

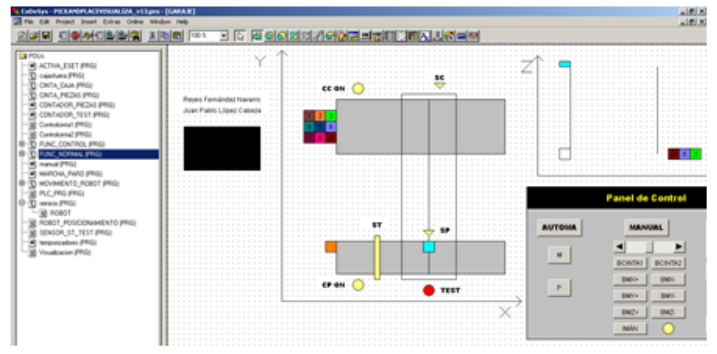

Figura 1. Ejemplo de un proyecto de Codesys. El alumno debe diseñar un sistema y el sistema de control de forma independiente aprovechando las herramientas de simulación y visualización de Codesys.

Cada curso se publican tres fechas. La primera es la fecha límite en la que los alumnos se organizan en grupos. Una vez se dispone del listado de grupos, se asigna un profesor tutor a cada grupo y se indica una fecha límite en la que cada grupo debe preparar una propuesta de problema a resolver y atender a una reunión con su tutor para discutir si el la propuesta es adecuada. Finalmente, antes de la presentación oral, cada grupo debe presentar la solución final a su tutor. La evaluación por parte del profesorado se realiza en base a una rúbrica para conseguir una valoración homogénea de todos los grupos, ya que cada profesor sólo evalúa a sus alumnos.

En el proyecto los alumnos deben proponer un problema de control describiendo el sistema dinámico a controlar, los sensores, los actuadores y las especificaciones de control. Una vez definido el problema deben programar en Codesys un simulador del sistema dinámico con sus actuadores y sensores, una visualización esquemática del mismo, el controlador y una visualización esquemática de su interfaz hombre máquina.

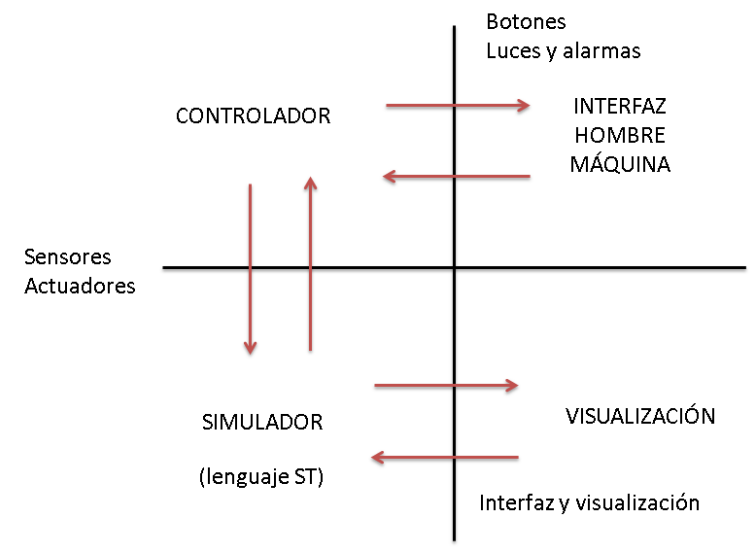

Figura 2. Elementos de código de un alumno y flujo de información.

\section{DESCRIPCIÓN DE LA EXPERIENCIA}

La experiencia está siendo muy positiva, aunque es importante hacer notar que es posible llevarla a cabo por el alto grado de implicación de todos los profesores de la asignatura, en particular, de los profesores de prácticas. Las asignaturas de "Automatización y control de sistemas de producción" y "Automatización Industrial" tienen unos 160 alumnos divididos en dos grupos. Cada grupo lo imparte un profesor (con 3.5 créditos de teoría y 2 créditos de prácticas en PAP), pero además, participan otros 4 profesores de prácticas (con 2 créditos en PAP cada uno) los cuales llevan a cabo labores de tutorización de trabajos. En media, cada año se presentan unos 60 proyectos en cada asignatura, repartidos a 10 trabajos por cada uno de los 6 tutores (4 profesores de prácticas y 2 de teoría). Esto implica el seguimiento de unos 30 alumnos. Este número es alto pero aceptable. Durante las semanas de la primera tutoría en la que se presentan los proyectos y en las semanas previas a la entrega, los profesores implicados tienen una gran carga de horas de atención a esos alumnos. Sin embargo, los profesores que han formado parte de la experiencia 
en general la valoran de forma muy positiva, ya que las tutorías derivadas de la realización de un proyecto permiten una interacción muy interesante con los alumnos, y 30 alumnos es un número manejable. A lo largo de los últimos años, hemos pensado en diferentes actividades. La metodología descrita es el resultado de muchos experimentos, y conseguir que la carga de trabajo fuera aceptable para poder implicar a la mayor cantidad de profesores ha sido un criterio determinante.

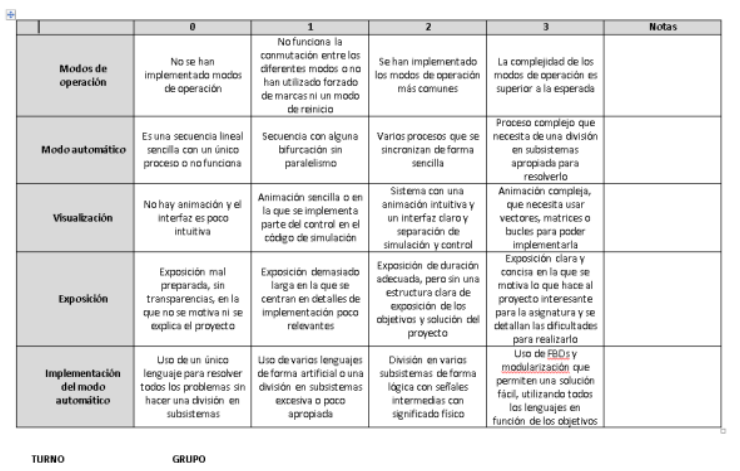

Figura 3. Rúbrica de evaluación de los proyectos.

Otro punto importante es el punto de vista organizativo. La evaluación del proyecto debe de ser repetible y compatible con las restricciones de la ETSI. El primer curso la evaluación se realizó en un día diferente de la convocatoria oficial. Esto conlleva toda una serie de problemas, sobre todo en la segunda y tercera convocatoria, complicando la gestión de posibles excepciones y restricciones de los alumnos. Para evitar problemas de fechas, se decidió que la entrega y defensa de los proyectos se realiza el mimo día del examen escrito. Esto implica que la evaluación de todos los proyectos de debe hacer en un único día. Hay una media de 60 proyectos en la primera convocatoria (en las otras el número es mucho menor, ya que por lo general sólo se presentan proyectos aptos para aprobar gracias al proceso de tutorización). A cada proyecto se le asignan 15 minutos para la defensa y las preguntas, lo que supone que son 15 horas de evaluaciones orales. Para poder hacerlo de forma práctica, las defensas se realizan en paralelo en 6 aulas diferentes, una por tutor. Normalmente el proceso de evaluación se extiende durante unas 2 o 3 horas, dependiendo del número de grupos que se presentan. Habitualmente, sólo realizan la presentación aquellos grupos que tienen un proyecto apto que ya han presentado a su tutor. Los alumnos que no se presentan en la primera convocatoria no presentan el proyecto. De nuevo la implicación de los profesores de prácticas es de vital importancia, ya que sin poder realizar las presentaciones en paralelo, no sería posible realizar la evaluación el mismo día que el examen escrito, dificultando todo el proceso.
Desde el punto de vista del alumno, después de 3 años, la única crítica recibida es la de aumentar el peso del proyecto en la nota final (o incluso eliminar la nota del examen). Los alumnos dedican una gran cantidad de horas a desarrollar su proyecto, sin embargo, parte del trabajo de tutorización es garantizar que la complejidad y alcance del proyecto son adecuados, en particular se intenta que los alumnos resuelvan un problema de automatización de una complejidad similar o incluso mayor que los problemas propuestos en los exámenes escritos. Al igual que en otras asignaturas de programación, resolver un problema en papel es más sencillo que resolverlo en una plataforma real y que funcione, pero por lo general, los alumnos que realizan un buen proyecto, tienen un dominio de la asignatura que les facilita estudiar y aprobar el examen escrito. De hecho son pocos los casos en los que un alumno ha realizado un buen proyecto, y ha suspendido el examen.

La metodología tiene por supuesto algunos errores. En particular, no hay una verificación del reparto de trabajo entre los diferentes miembros de los grupos, lo que posibilita a los alumnos evitar trabajar en el proyecto si encuentran compañeros que les incluyan en sus grupos. Con el ratio de alumnos/profesor actual, consideramos que no tenemos ninguna metodología con una carga de trabajo aceptable para superar este problema, por lo que se mantiene como requisito para aprobar la asignatura, aprobar el examen escrito. La complejidad de los problemas de los exámenes es similar a la de los proyectos, por lo que los alumnos que no han trabajado el proyecto suelen tener dificultades en resolverlos.

\section{RESULTADOS}

La metodología propuesta de evaluación de proyectos de automatización ha sido un éxito en las dos asignaturas presentadas. Adicionalmente se ha utilizado una idea similar basada en enunciados diseñados por alumno, tutorización y defensa oral en otras asignaturas como "Control y programación de robots manipuladores" y "Proyectos de automatización" usando otras plataformas de programación. La realidad es que requiere una gran implicación tanto de los profesores, como un mayor trabajo de los alumnos, sin embargo la realimentación es muy buena.

Los alumnos valoran muy positivamente trabajar con una plataforma industrial (aunque sea en simulación) $\mathrm{y}$ aprender de forma autónoma en base a un proyecto complejo. La libertad de diseñar sus propios problemas es muy motivadora para algunos alumnos, y todos los años nos encontramos con grupos cuyo proyecto excede los objetivos de la asignatura. Finalmente, cabe destacar que el número de alumnos 
que nos contactan para realizar trabajos de fin de grado o de master relacionados con la asignatura de automatización aumenta cada año.

Desde el punto de vista docente, la asignatura tiene un porcentaje de aprobados alto, pero además consideramos que el nivel de los alumnos ha mejorado como demuestran las soluciones de los alumnos en los exámenes escritos, que presentan problemas complejos de solución abierta.

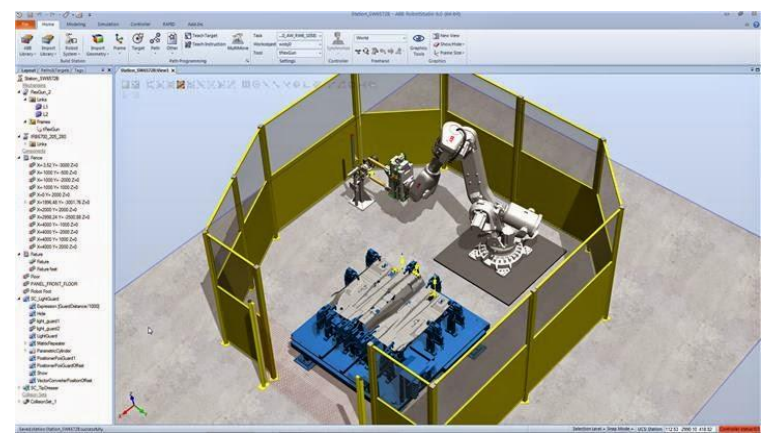

Figura 4. Ejemplo de un proyecto de Robotstudio. El alumno debe diseñar un sistema y programar el comportamiento del robot aprovechando las herramientas de simulación y visualización de Robotstudio.

Como conclusión final, los resultados de la experiencia demuestran que es posible obtener buenos resultados de aprendizaje si los alumnos realizan actividades diferentes a asistir a clases magistrales o realizar exámenes escritos tal y como defiende el proceso de Bolonia, sin embargo es necesaria una buena planificación que tenga en cuenta las restricciones impuestas por los planes de estudio actuales en relación al ratio de alumnos/profesor, sistemas de evaluación y horas de dedicación. Esto supone un gran esfuerzo e implicación por parte del profesorado.

\section{Agradecimientos}

Esta experiencia no hubiera sido posible sin el apoyo de Miguel Ángel Ridao, Ascensión Zafra, Daniel Limón, Ignacio Alvarado, Teodoro Álamo, Fernando Castaño, Mario Pereira, José Enrique Alonso y Francisco Gordillo.

\section{English summary}

\section{EVALUATION OF PROJECTS IN AUTOMATION AND ROBOTICS COURSES}

\section{Abstract}

This paper presents the implementation of a system for evaluating projects in various subjects of our department related to automation and robotics. The proposed teaching is based on the realization by students of an open group automation project, in which they not only have to design the solution, but also the problem. The open nature of the project and the large number of students motivated the distribution of the different groups among the different teachers of the subject. Each group of students has at least two meetings with their teacher to agree on the problem to be solved in order to adapt the complexity and scope to the objectives of the subject. The evaluation of the projects is done through an oral presentation on the same day as the written examination of the official calls. The proposed methodology has been implemented with successes in various subjects and more than 600 students have participated in recent years with great acceptance by both teachers and students.

Keywords: Evaluation, automation and robotics

\section{Referencias}

[1] Ridao M.A. (2016), "Introducción a la programación de autómatas programables usando CoDeSys", Editorial Universidad de Sevilla, España.

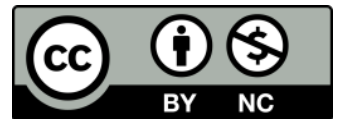

(C) 2018 by the authors. Submitted for possible open access publication under the terms and conditions of the Creative Commons Attribution CC-BY-NC 3.0 license (https://creativecommons.org/licenses/by-nc/3.0). 